\title{
DISCOVERY OF A GAS GIANT PLANET IN MICROLENSING EVENT OGLE-2014-BLG-1760
}

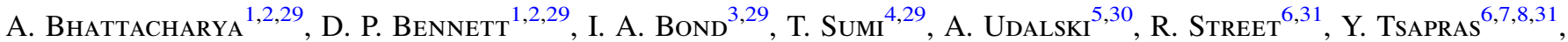

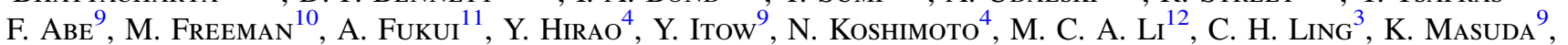 \\ Y. Matsubara $^{9}$, Y. Muraki ${ }^{9}$, M. Nagakane ${ }^{4}$, K. Ohnishi ${ }^{13}$, N. Rattenbury ${ }^{12}$, T. Saito ${ }^{14}$, A. Sharan ${ }^{12}$, D. J. Sullivan ${ }^{15}$, \\ D. SUZUKI ${ }^{2}$, P. J. Tristram ${ }^{16}$ \\ (MOA COllaboration), \\ J. Skowron ${ }^{5}$, M. K. Szymański ${ }^{5}$, I. Soszyński ${ }^{5}$, R. Poleski ${ }^{5,17}$, P. Mróz ${ }^{5}$, S. Kozlowski ${ }^{5}$, P. Pietrukowicz ${ }^{5}$, K. UlaCzyK ${ }^{5}$, \\ L. WYRZYKOWSKI ${ }^{5}$ \\ (OGLE COLlaboration),
}

AND

E. Bachelet ${ }^{6,18}$, D. M. Bramich ${ }^{18}$, G. D’Ago ${ }^{19,20}$, M. DominiK ${ }^{21,32}$, R. Figuera Jaimes ${ }^{21,22}$, K. Horne $^{21}$,

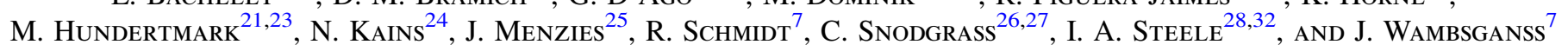
(ROBONET COLlaboration)

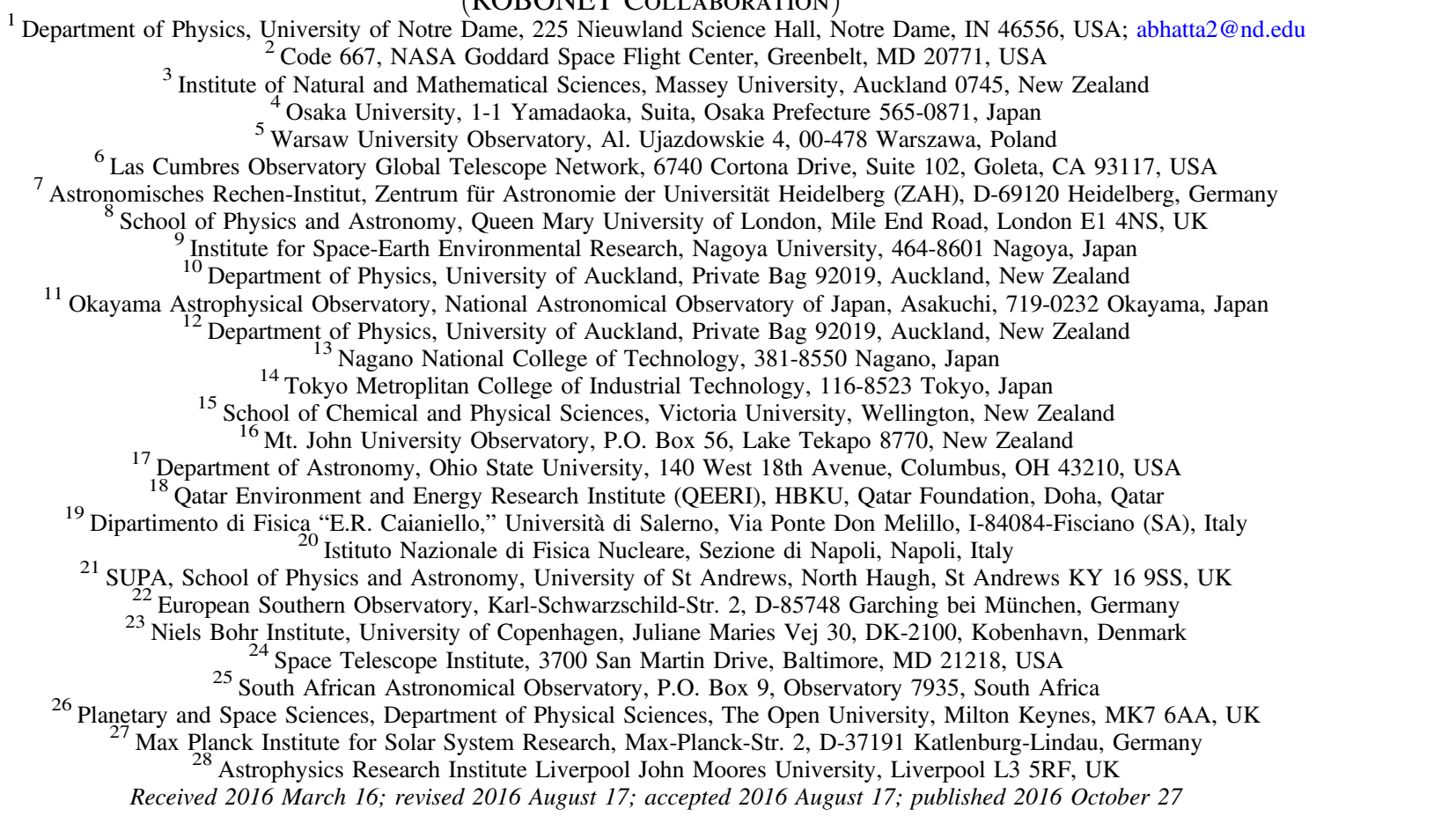

\section{ABSTRACT}

We present the analysis of the planetary microlensing event OGLE-2014-BLG-1760, which shows a strong lightcurve signal due to the presence of a Jupiter mass ratio planet. One unusual feature of this event is that the source star is quite blue, with $V-I=1.48 \pm 0.08$. This is marginally consistent with a source star in the Galactic bulge, but it could possibly indicate a young source star on the far side of the disk. Assuming a bulge source, we perform a Bayesian analysis assuming a standard Galactic model, and this indicates that the planetary system resides in or near the Galactic bulge at $D_{L}=6.9 \pm 1.1 \mathrm{kpc}$. It also indicates a host-star mass of $M_{*}=0.51_{-0.28}^{+0.44} M_{\odot}$, a planet mass of $m_{\mathrm{p}}=0.56_{-0.26}^{+0.34} M_{\mathrm{J}}$, and a projected star-planet separation of $a_{\perp}=1.75_{-0.33}^{+0.34}$ au. The lens-source relative proper motion is $\mu_{\text {rel }}=6.5 \pm 1.1$ mas yr$^{-1}$. The lens (and stellar host star) is estimated to be very faint compared to the source star, so it is most likely that it can be detected only when the lens and source stars start to separate. Due to the relatively high relative proper motion, the lens and source will be resolved to about $\sim 46$ mas in $6-8 \mathrm{yr}$ after the peak magnification. So, by 2020-2022, we can hope to detect the lens star with deep, high-resolution images.

Key words: gravitational lensing: micro - planetary systems

\footnotetext{
29 Microlensing Observation in Astrophysics.

${ }^{30}$ Optical Gravitational Lensing Experiment.

31 Robonet.

32 Royal Society University Research Fellow.
} 


\section{INTRODUCTION}

Gravitational microlensing is one of the popular techniques for detecting exoplanets. It is unique in its ability to detect planets (Gould \& Loeb 1992) just outside of the snow line (Lissauer 1993) down to an Earth mass (Bennett \& Rhie 1996), which is difficult or impossible with other methods. According to the core accretion theory, the snow line (Kenyon \& Hartmann 1995; Ida \& Lin 2005; Kennedy et al. 2006; Lecar et al. 2006) plays a very crucial role in the planet formation process. Beyond the snow line, ices condense, increasing the density of the solid materials. Higher density of solids speeds the planet formation process in the protoplanetary disk, hence forming cold planets quickly beyond the snow line. The planets, discovered by microlensing, provide the statistics needed to understand the architecture of cold planets beyond the snow line (Gould et al. 2010b; Cassan et al. 2012; Shvartzvald et al. 2016; Suzuki et al. 2016). Since this method does not rely on the light from the host stars, it can detect planets, even when the host stars cannot be detected (Mao \& Paczyński 1991). Most of the planets discovered so far using microlensing are $\sim 1-8 \mathrm{kpc}$ away. Thus, microlensing is able to detect planets in the inner Galactic disk and bulge, where it is difficult to detect planets with other methods. Thus, microlensing has the potential to measure how the properties of exoplanets depend on the Galactic environment.

For most planetary microlensing events, the angular Einstein radius, $\theta_{E}$, is measured from the finite source effect. In events where the lens-star brightness (Bennett et al. 2006) or the parallax effect is measured (Gould 1992; Gaudi et al. 2008; Muraki et al. 2011), the planetary mass and distance to the planetary system can be determined. Hence, this technique can be used to build statistics of the planetary mass as a function of the host-star mass. Since the planets detected by microlensing are $\sim 1-8 \mathrm{kpc}$ away, the distance to the planetary system will also allow the determination of the planetary mass function as a function of the distance toward the Galactic center.

In this paper we present the discovery of a gas giant planet orbiting the lens stars for microlensing event OGLE-2014BLG-1760. The mass ratio of this planet is $q=8.64 \times 10^{-4}$, which is slightly less than the Jupiter/Sun mass ratio. The paper is organized as follows: Section 2 describes the lightcurve data collected for the event OGLE-2014-BLG-1760. The next section (Section 3) is divided into four parts: Section 3.1 summarizes data reduction procedures for the different data sets, Section 3.2 shows the best-fit model and procedures that are used to obtain it, Section 3.3 describes how the limb darkening of the source is modeled, and Section 3.4 presents our attempt to detect the microlensing parallax effect in the light curve. In Section 4, we discuss source brightness and angular radius measurement and derive the lens-source relative proper motion. Section 5 discusses an estimate of the lens properties and the future possible investigations.

\section{OBSERVATIONS}

The Optical Gravitational Lensing Experiment (OGLE) Collaboration operates a microlensing survey toward the Galactic bulge, with the $1.3 \mathrm{~m}$ Warsaw telescope from Las Campanas Observatory in Chile. Most of the OGLE-IV (phase 4) observations were taken in the Cousins $I$ band, with occasional observations in Johnson $V$ band (Udalski et al. 2015). In the year 2014, OGLE Early Warning System
(EWS) (Udalski et al. 1994) detected 2049 microlensing candidates, of which event OGLE-2014-BLG-1760 was the 1760th one.

The Microlensing Observation in Astrophysics (MOA; Bond et al. 2001) Collaboration also operates a microlensing survey toward the Galactic bulge with the $1.8 \mathrm{~m}$ MOA-II telescope from Mount John Observatory at Lake Tekapo, New Zealand. The observations are mostly taken in the MOA-red wide-band field, which covers the wavelengths of the standard Cousins $R$ $+I$ bands. For year 2014, MOA reported $\sim 33$ microlensing anomalies, including the OGLE-2014-BLG-1760 planetary event.

Both of these telescopes have relatively large fields of view, $2.2 \mathrm{deg}^{2}$ for MOA-II and $1.4 \mathrm{deg}^{2}$ for OGLE-IV. These enable survey observations with cadences as high as one observation every 15 minutes, and this allows the surveys to detect the sharp light-curve features of planetary light curves, when they are only smoothed by the finite source effects of a mainsequence source star. It is the high-cadence observation of microlensing events of the MOA-II and OGLE-IV surveys that helps in detecting microlensing anomalies, including the microlensing planetary signatures.

The microlensing event OGLE-2014-BLG-1760 was discovered at (R.A., decl.) $(2000)=(17: 57: 38.16,-28: 57: 47.37)$ $((l, b)=(1.3186,-2.2746))$ by the OGLE EWS on 2014 August 22 around 7.25 a.m. EDT. The same event was detected by MOA on 2014 August 31 as MOA-2014-BLG-547. Later, on 2014 September 10, the MOA Collaboration detected the planetary cusp crossing and announced the anomaly in the light curve (around HJD $-2,450,000=6911$ in Figure 1). In response to the MOA anomaly alert, the follow-up groups Robonet and $\mu \mathrm{FUN}$ started collecting data on this event. The high-cadence observation of the MOA-II survey covered most of the cusp crossing, and the trough after the cusp crossing was well covered by follow-up groups, Robonet and $\mu$ FUN. The Robonet group observed the event with $1 \mathrm{~m}$ robotic telescopes at Cape Town, South Africa, and at Siding Springs, Australia, in the Sloan $I$ band. The $\mu$ FUN group also observed the event with the $1.3 \mathrm{~m}$ SMARTS CTIO telescopes in both the $V$ and $I$ bands. All these observations are shown in Figure 1. Although the $\mu$ FUN data are pretty flat because the trough is followed by the normal single-lens decrease (see Figure 1), the $\mu$ FUN data do help to exclude the wide model with $s=1.27$ (discussed in Section 3.2). It is true that the constraint from the Robonet and OGLE data is stronger because of better Robonet coverage and OGLE observations over a wider range of magnifications. $\mu$ FUN also took a few data in $H$ band that are not used in this analysis but later, along with follow-up observations, can be used to constrain the source color. We are grateful to Andy Gould and the $\mu \mathrm{FUN}$ team for allowing us to use their data.

\section{DATA REDUCTION AND MODELING}

\subsection{Data Reduction}

Photometric reductions of all the images were done using the difference image method (Tomany \& Crotts 1996; Alard \& Lupton 1998; Alard 2000). The MOA images were reduced using the MOA difference image analysis pipeline (Bond et al. 2001). The data were then detrended using the non-2014 data to minimize the error due to the effects of differential refraction, seeing, and airmass. To avoid systematic errors due to flat-field changes and changes in detector sensitivity, we 


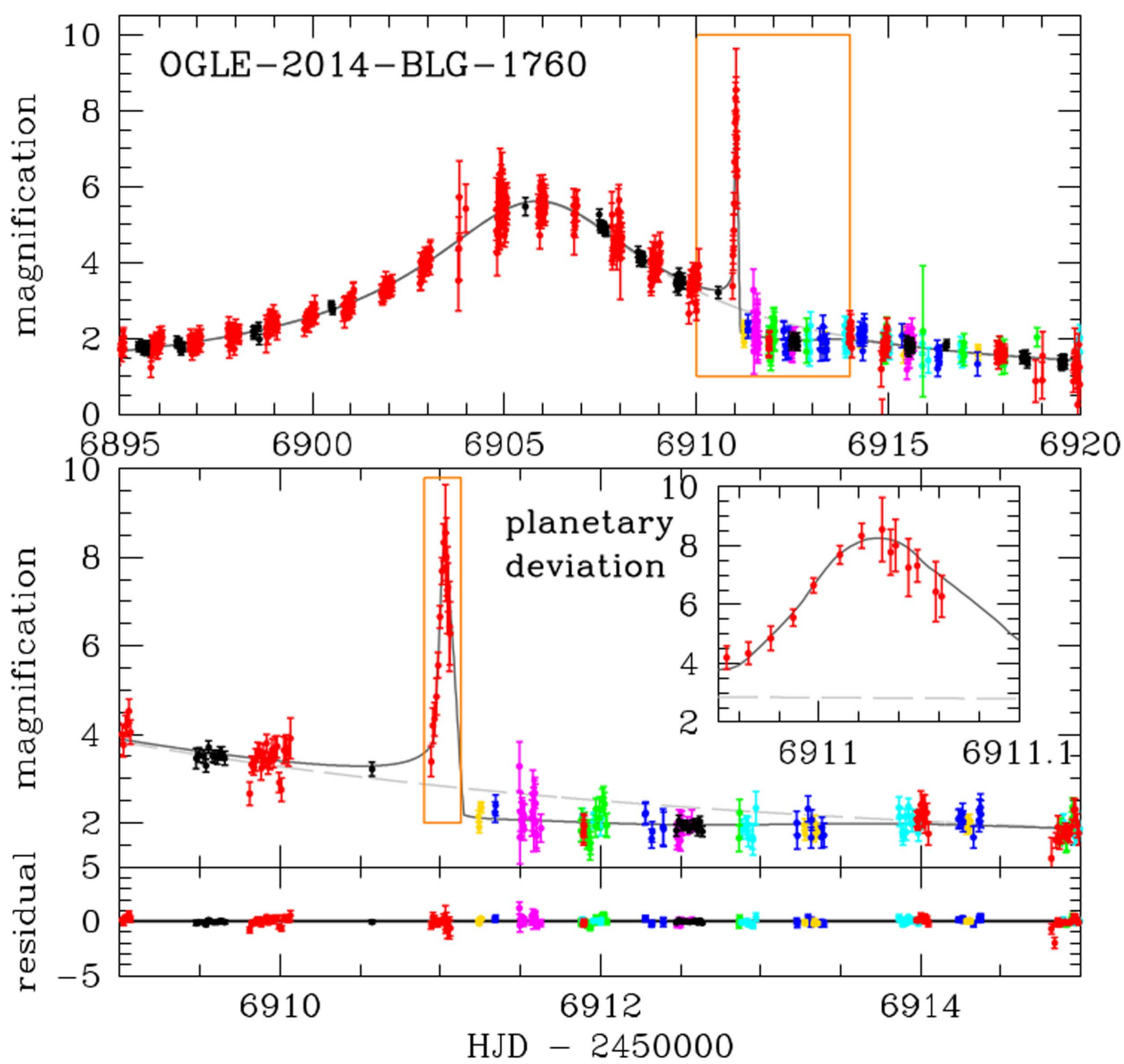

Figure 1. Light curve of event OGLE-2014-BLG-1760. The light-gray dashed line represents the best-fit single-lens model, and the dark-gray line represents the best planetary model. The data are plotted in the following colors: red for the MOA-red band, black for OGLEI, purple for OGLE $V$, (difficult to see due to overlap with the OGLE $I$ data), blue for the ROBONET SAAO-A $I$ band, gold for the ROBONET SAAO-B $I$ band, green for the ROBONET Siding Spring-A $I$ band, cyan for the ROBONET Siding Spring-B $I$ band, and magenta for the $\mu$ FUN $I$ band.

removed data prior to 2011 and only used the data after HJD $-2,450,000=5596$ (2011 February) for modeling. OGLE data were reduced using the OGLE Difference Image Analysis software and optimal centroid method (Wozniak 2000; Udalski 2003). The $\mu$ FUN photometry was produced using a modified version of the PySIS package (Albrow et al. 2009). Robonet data were reduced using the DanDIA package (Bramich 2008).

The error-bar estimates obtained from the photometry codes are sufficient to find the best-fit model. These error-bar estimates are good measures of the relative uncertainties for measurements with the same telescope, but they are often wrong by a factor of $\sim 2$ (Yee et al. 2012). To determine the uncertainties for the model parameters, it is necessary to have accurate error bars that will give $\chi^{2} /$ dof $\approx 1$ for each data set. We used the method of Bennett et al. (2014) to normalize the error bars using $\sigma_{\mathrm{i}}^{\prime}=k \sqrt{\sigma_{\mathrm{i}}^{2}+e_{\min }^{2}}$. Here $\sigma_{\mathrm{i}}$ is the initial uncertainty of the $i$ th data point; $\sigma_{i}^{\prime}$ is the modified error bar. A $k$ value is selected for each data set to give $\chi^{2} /$ dof $=1$. Our initial fits used $k=1.50$ and $e_{\min }=0.003$ for all data sets. Next, once the best-fit model was found, the values of $k$ were modified for each data set to satisfy $\chi^{2} /$ dof $=1$. The number of data points used for each telescope and their corresponding $k$ values are listed in Table 1 . The modification in $e_{\min }$ value showed a negligible change in best-fit value. New fits were done with the new error bars, which resulted in very small changes to the model parameters.
Table 1

Data Reduction Summary

\begin{tabular}{lccc}
\hline \hline Telescope & Filter & $N^{\mathrm{a}} / N^{\mathrm{b}}$ & $k$ \\
\hline MOA & Red $(I+R)$ & $11,655 / 11,673$ & 0.63 \\
OGLE & $I$ & $10,779 / 10,794$ & 0.98 \\
OGLE & $V$ & $119 / 119$ & 0.84 \\
$\mu$ FUN & $I$ & $45 / 45$ & 0.79 \\
$\mu$ FUN & $V$ & $3 / 4$ & 0.99 \\
ROBONET SAAO-A A & $I$ & $54 / 54$ & 1.16 \\
ROBONET SAAO-B B & $I$ & $32 / 32$ & 0.96 \\
ROBONET Siding Spring-A A & $I$ & $56 / 56$ & 1.24 \\
ROBONET Siding Spring-B B & $I$ & $75 / 75$ & 1.23 \\
\hline
\end{tabular}

Notes.

${ }^{\text {a }}$ Number of data points used.

b Number of total observations; MOA and OGLE data points before HJD $-2,450,000=5596$ were excluded due to systematic errors, as mentioned in Section 3.1 .

\subsection{Best-fit Model}

We begin our modeling of the OGLE-2014-BLG-1760 light curve with a single-lens microlensing model (Paczyński et al. 1986). There are three nonlinear model parameters for a singlelens event: $t_{0}$, the time of peak magnification; $u_{0}$, the minimum separation between source and lens in Einstein radius units; and 
Table 2

Model Parameters

\begin{tabular}{lccc}
\hline \hline Parameter & Units & Binary-lens Best Fit & Single-lens Best Fit \\
\hline$t_{\mathrm{E}}$ & days & $15.87 \pm 0.41$ & 15.47 \\
$t_{0}$ & HJD $-2,450,000$ & $6905.856 \pm 0.026$ & 6905.9541 \\
$u_{0}$ & & $0.1806 \pm 0.0074$ & 0.19 \\
$s$ & & $0.8269 \pm 0.0047$ & \\
$\theta$ & radians & $-0.3977 \pm 0.0086$ & \\
$q$ & $10^{-4}$ & $8.64 \pm 0.89$ & \\
$t_{\star}$ & days & $0.0366 \pm 0.0044$ & 24036.80 \\
fit $\chi^{2}$ & & 22818.05 & \\
\hline
\end{tabular}

$t_{\mathrm{E}}$, the Einstein radius crossing time. There are also two linear parameters for each data set: the source flux, $f_{\mathrm{s}}$, and the blend flux, $f_{\mathrm{bl}}$. We find the best single-lens parameters as the starting point for a systematic search through parameter space to find the best binary-lens solution. Because the light curve follows a single-lens shape for most of its history, except in the vicinity of the planetary feature at HJD $-2,450,000 \approx 6911$, we can use the best-fit single-lens parameters as the starting point for an initial condition grid search, following Bennett (2010).

To describe a binary lens, we need three additional parameters: the lens mass ratio, $q$; the projected separation between the lens masses, $s$, measured in Einstein radius units; and the angle between the source trajectory and the lens axis, $\theta$. Also, binary events often have caustic or cusp crossings, which resolve the angular size of the source, so we need an additional parameter, the source radius crossing time, $t_{*}$, to model finite source effects. With the single-lens parameters fixed to the best-fit values, we used the initial condition grid search method to search over the parameter ranges $0.48 \leqslant s \leqslant 2.10$, $-4 \leqslant \log q \leqslant-2$, and $-\pi \leqslant \theta \leqslant \pi$, with $t_{*}$ fixed at $t_{*}=0.05$. We then select $\sim 10$ of the best-fit values from the initial condition grid search (with very different values of $q, s$, and $\theta$ ) to use as initial conditions for full, nonlinear modeling runs using the Bennett (2010) $\chi^{2}$ minimization recipe, which is a modification of the Markov Chain Monte Carlo algorithm (Verde et al. 2003). The parameters of the best-fit binary-lens model and the $\chi^{2}$ improvement are compared with the best-fit single-lens model in Table 2. The uncertainties on the binarylens model parameters were calculated by taking an average over the MCMC chains of the fits. The binary-lens best-fit model has $q=8.64 \times 10^{-4}$ and improves the renormalized $\chi^{2}$ by $\Delta \chi^{2}=1218.75$ compared to the best-fit single-lens model. Hence, the binary-lens model is the preferred model.

High-magnification planetary microlensing events usually have a "close-wide" degeneracy, in that solutions with $s \leftrightarrow 1 / s$ are nearly degenerate. This is usually not the case for low-magnification planetary events because the caustic structure for the major and minor image caustics is quite different. However, the major and minor image caustics with $s \leftrightarrow 1 / s$ are encountered at the same single-lens magnification, and with specific source trajectories, it is possible to produce similar light curves with both $s>1$ and $s<1$, particularly if the light curves are not very well sampled. Therefore, we have searched for models with $s \sim 1 / 0.83=1.20$. We found a best-fit $s>1$ model with $s=1.27$, but this is a worse fit than the best-fit model (with $s=0.83$ ) by $\Delta \chi^{2} \sim 215$. This is because the OGLE-2014-BLG-1760 is well sampled, so the $s=1.27$ model is excluded, and the $s=0.83$ is the only viable solution. The best fit model and its corresponding caustic crossing structure are shown in Figures 1 and 2, respectively.

\subsection{Limb-darkening Effect}

The photometric calibrations and the extinction toward the red clump stars in the vicinity of the source are discussed in Section 4. The extinction-corrected color of the source is $(V-I)_{\mathrm{S}, 0}=0.34$, as discussed in Section 4. This color implies that the source is an $\sim$ A9 star with $T_{\text {eff }} \sim 7352 \mathrm{~K}$ from Kenyon \& Hartmann (1995). We used a linear limbdarkening model, and from Claret (2000), we selected the limbdarkening parameters $u$ to be $0.4204,0.5790$, and 0.46035 , corresponding to the $V, I$, and MOA-red bands, respectively. These correspond to a temperature of $T_{\text {eff }}=7352 \mathrm{~K}$ and a surface gravity of $\log g=4.5$. The parameters of the best-fit model were insensitive to the precise limb-darkening parameters.

\subsection{Search for a Microlensing Parallax Signal}

The microlensing parallax effect has been detected in a number of planetary microlensing events (Gaudi et al. 2008; Bennett et al. 2010; Muraki et al. 2011), where it has allowed the lens system masses to be measured. Due to the Earth's orbital motion about the Sun, the apparent lens-source relative motion deviates from uniform linear motion. This phenomenon is known as the microlensing parallax effect (Gould 1992; Alcock et al. 1995). The parallax effect can be described with the parallax vector $\pi_{\mathrm{E}}=\left(\pi_{\mathrm{E}, \mathrm{N}}, \pi_{E, E}\right)$, where the direction of $\pi_{\mathrm{E}}$ is the same as the lens-source relative proper motion, $\mu_{\text {rel }}$. The amplitude of $\pi_{\mathrm{E}}$ is the inverse of the Einstein radius projected to the observer's plane, $\pi_{\mathrm{E}}=\mathrm{au} / \tilde{r}_{\mathrm{E}}$. When both the microlensing parallax effect and the angular Einstein radius (as described in Section 4) are measured, we can determine the lens system mass and distance from the following equations:

$$
\begin{gathered}
M_{\mathrm{L}}=\frac{\theta_{\mathrm{E}}}{k \pi_{\mathrm{E}}} \\
D_{\mathrm{L}}=\frac{\mathrm{au}}{\pi_{\mathrm{E}} \theta_{\mathrm{E}}+\frac{\mathrm{au}}{D_{\mathrm{S}}}},
\end{gathered}
$$

where $k=4 G /\left(c^{2} \mathrm{au}\right)=8.14$ mas $M_{\odot}^{-1}, M_{\mathrm{L}}$ is the total mass of the host star and planet, and $D_{\mathrm{L}}$ and $D_{\mathrm{S}} \sim 8 \mathrm{kpc}$ are the distances to the lens system and source stars, respectively. The host-star mass, $M_{*}$, is given by $M_{*}=M_{\mathrm{L}} /(1+q)$.

In order to include the microlensing parallax effect, we must add two new model parameters $\pi_{\mathrm{E}}$ and $\phi_{\mathrm{E}}$, the magnitude and direction angle of the parallax vector, respectively. (The north and east components of $\pi_{\mathrm{E}}$ are given by $\pi_{\mathrm{E}, \mathrm{N}}=\pi_{\mathrm{E}} \cos \left(\phi_{\mathrm{E}}\right)$ and $\pi_{E, E}=\pi_{\mathrm{E}} \sin \left(\phi_{\mathrm{E}}\right)$, respectively.) Our best-fit parallax model has an unusually large $\pi_{\mathrm{E}}$ value of $\pi_{\mathrm{E}}=5.86$, which would imply a very nearby and low-mass lens if it is an accurate measurement, but the improvement in $\chi^{2}$ is only $\Delta \chi^{2}=9.90$ over the best fit without parallax. We can examine the origins of this parallax signal by examining the cumulative $\Delta \chi^{2}$ between the nonparallax and parallax models as a function of time, which we show in Figure 3 . The cumulative $\Delta \chi^{2}$ is displayed for the MOA and OGLE data separately, and we can see that only the OGLE data favor the parallax model. Penny et al. (2016) consider the distribution of published planetary microlensing events and show that there are an implausibly large number with "high" microlensing parallax values 


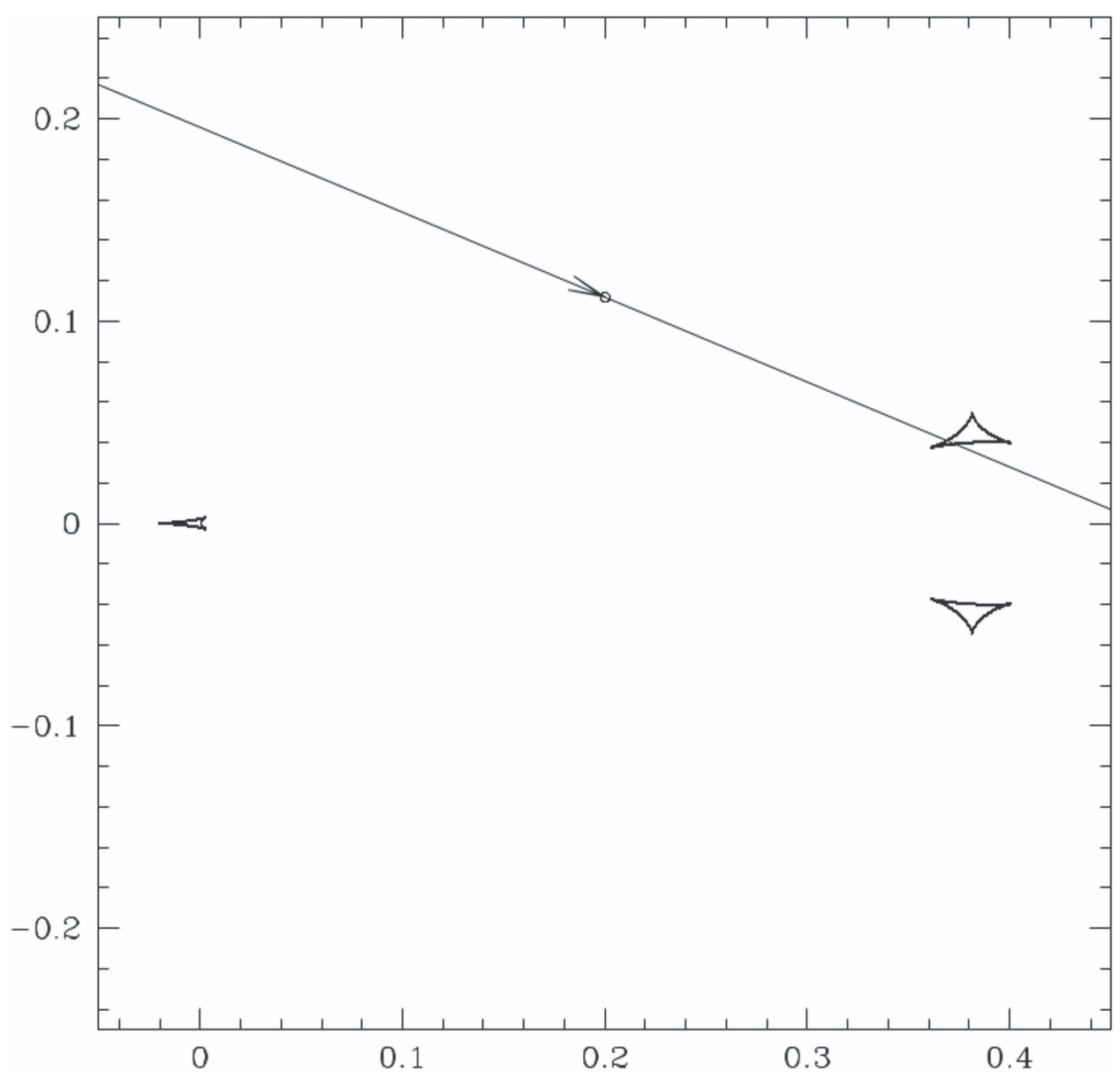

Figure 2. Caustic configuration and source trajectory for the best-fit planetary model. When the source crosses inside the planetary caustic, its magnification jumps as two new, highly magnified images are created, and then the magnification drops just as abruptly when the source exits and the two images disappear. The two caustic crossings are at HJD $-2,450,000=6911.00$ and 6911.07. Since the interval between the caustic crossings is similar to the source radius crossing time, $t_{*}$, the two magnification peaks merge into one peak, as shown in Figure 1 at HJD -2,450,000 6911. Also, when the source passes between the two minor image planetary caustics, the magnification of the source brightness drops, as can be seen in the light curve after caustic crossing, between HJD $-2,450,000=6911.20$ and 6914.00 in Figure 1.

$\left(\pi_{\mathrm{E}} \gtrsim 1\right)$. This suggests that some of the published planetary events have spurious large $\pi_{\mathrm{E}}$ values (Han et al. 2016). We conclude that this microlensing parallax measurement for OGLE-2014-BLG-1760 is also spurious.

\section{CALIBRATION AND SOURCE PROPERTIES}

The OGLE data were taken in the OGLE-IV $I$ and $V$ bands, which we calibrate to the OGLE-III catalog Cousins $I$ and Johnson $V$ band (Szymański et al. 2011a). A total of 151 bright $(I \leqslant 16.50)$ and isolated stars were matched in both passbands and used for this calibration. The following calibration relations are used to convert OGLE VI magnitudes to OGLE-III catalog Cousins $I$ and Johnson $V$ magnitude:

$$
\begin{aligned}
& I_{\mathrm{OGLE}_{\mathrm{IV}}}=-0.0471+0.99867 I_{\mathrm{OGLE}_{\mathrm{III}}}+0.00133 V_{\mathrm{OGLE}_{\mathrm{III}}} \\
& V_{\mathrm{OGLE}_{\mathrm{IV}}}=-0.3444-0.10068 I_{\mathrm{OGLE}_{\mathrm{III}}}+1.10068 V_{\mathrm{OGLE}_{\mathrm{III}}}
\end{aligned}
$$

The uncertainty in the brightness due to these calibration relations is $\sim 0.01 \mathrm{mag}$. The source brightness in the OGLE-III catalog scale is $I_{\mathrm{S}}=19.07 \pm 0.14, V_{\mathrm{S}}=20.51 \pm 0.26$. The errors in brightness are calculated from MCMC averages over all the MCMC fits and from the uncertainty in the calibration relations.

There is a single magnified OGLE $V$-band observation at HJD $-2,450,000=6896.50$, and this results in a relatively large $(10 \%)$ uncertainty in the $V$-band source flux for the bestfit model. As a result, the OGLE VI source gives a source-star color of $V-I=1.45 \pm 0.11$. The color of the source star is bluer than the average main-sequence-star color, as can be seen in Figure 4. Because we have only this single $V$-band measurement, we also calculate the source-star color from MOA-red and OGLEI band following Gould et al. (2010a) and Bennett et al. (2012). Since we have a large number of MOAred and OGLEI-band observations when the source is significantly magnified, we expect a robust measurement of the $R_{\mathrm{MOA}}-I$ source color. About 140 bright, isolated stars with $I$-band magnitude $I<16$ and $1.0<V-I<2.6$ are matched between the MOA-II and OGLE-III reference images, and we used these to find the following calibration relation:

$$
R_{\mathrm{MOA}}-I_{\mathrm{OGLE}_{\mathrm{III}}}=0.18143 \times(V-I)_{\mathrm{OGLE}_{\mathrm{III}}},
$$

where the $R_{\mathrm{MOA}}$ passband has been calibrated to give $R_{\mathrm{MOA}}=I_{\mathrm{OGLE}_{\mathrm{III}}}$ when $(V-I)_{\mathrm{OGLE}_{\mathrm{III}}}=0$. The rms error for this calibration relation is 0.0343 , for a formal uncertainty of 


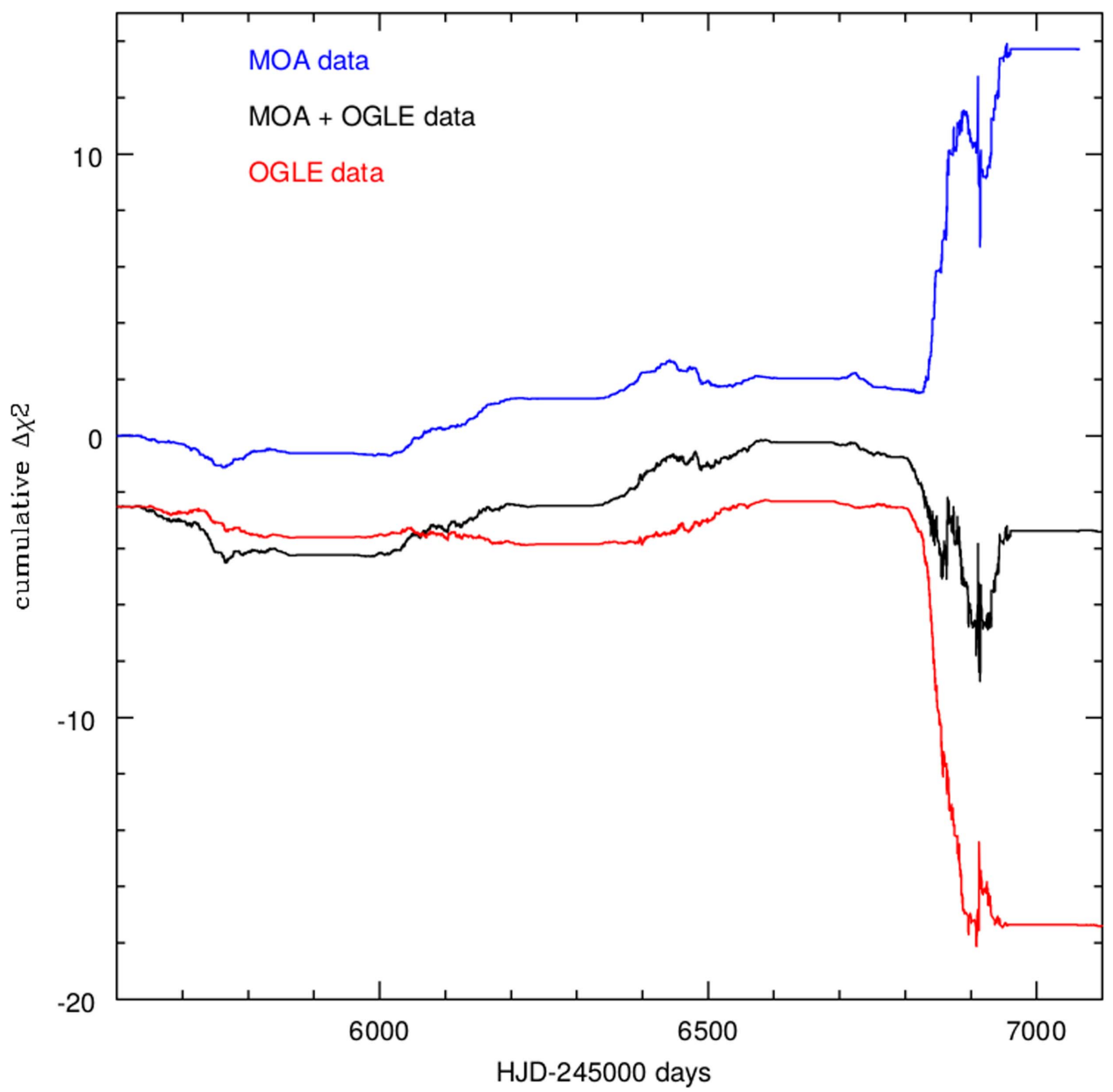

Figure 3. Difference in the cumulative $\chi^{2}$ values between the best-fit models with and without parallax for MOA data (blue), OGLE data (red), and total MOA + OGLE data (black). The negative cumulative $\Delta \chi^{2}$ means that the parallax model is supported. Also, MOA data do not support parallax, whereas OGLE data support the parallax model. The $\chi^{2}$ improvement from MOA + OGLE data for the parallax model is $\sim 3.60$. The overall $\chi^{2}$ improvement for the parallax model from all the telescopes is $\sim 9.90$. Hence, the parallax signal is dubious and probably a false signal.

$0.0343 / \sqrt{N}=0.0029$, but we assume a calibration uncertainty of 0.02 for $R_{\mathrm{MOA}}-I_{\mathrm{OGLE}_{\mathrm{II}}}$.

Our models give source brightnesses of $I_{S}=19.07 \pm 0.14$ and $R_{\mathrm{MOA}, S}=19.34 \pm 0.15$ and a calibrated source color of $V-I=1.52 \pm 0.11$. This color is less than $1 \sigma$ away from the color of the source derived from OGLE $V$-band data, so we conclude that the blue color from the OGLE data is probably real. However, the average color from both measurements is $V-I=1.48 \pm 0.08$, which still has a relatively large error bar. We take this average as the correct color of the source. The green points in the color-magnitude diagram (CMD) depicted by Figure 4 show the Hubble Space Telescope (HST) CMD plot (Holtzman et al. 1998) shifted to have the same red clump centroid position as the OGLE-III stars within $140^{\prime \prime}$ of the source star, and the cyan point shows the color of the source. The source color is slightly bluer than the main-sequence-star distribution, shown with green points in the HST CMD in Figure 4 . The average color of main-sequence bulge stars in the same magnitude range as the source $(I=19.07 \pm 0.14)$ in the $H S T$ CMD is $V-I=1.75$. We can estimate the probability that a star with the measured color of the source $(V-I=1.48 \pm 0.08)$ is drawn from this HST distribution by integrating the Gaussian describing the measured source color with its error bar over the distribution of stars in the same magnitude range from the HST CMD. We then divide this result by the result of the integral with the same error bar, but centered on the average color of $V-I=1.75$. The ratio of the integral centered on the measured color and the integral centered on the average color is 0.05 , indicating that this source color is marginally consistent with being drawn from the known bulge-star population. With nearly 50 planetary microlensing events published to date, we would expect one or two to be found with such a blue source star. On the other hand, it is also possible that blue stars are preferentially lensed because they are at a greater distance. This can be the case if the source is a young blue (late A or early F) star on the far side of the Galactic disk, assuming negligible extinction beyond the bulge on this line of sight. Such stars would have a much larger microlensing event rate, so they could be preferentially lensed by bulge stars. It is also possible that the CMD in this field may be noticeably different than that of Baade's window where the Holtzman et al. (1998) image was taken.

We calculate the extinction in the direction of the source using the method described by Bennett et al. (2014). The position of the centroid of the red clump in the OGLE-III catalog is found to be $(V-I, I)_{\mathrm{RC}}=(2.20,15.84)$. From Bensby et al. (2011) and Nataf et al. (2013), the dereddened red clump centroid is determined to be $(V-I, I)_{\mathrm{RC}}=(1.06$, 14.39). Hence, the extinction at this Galactic coordinate is 


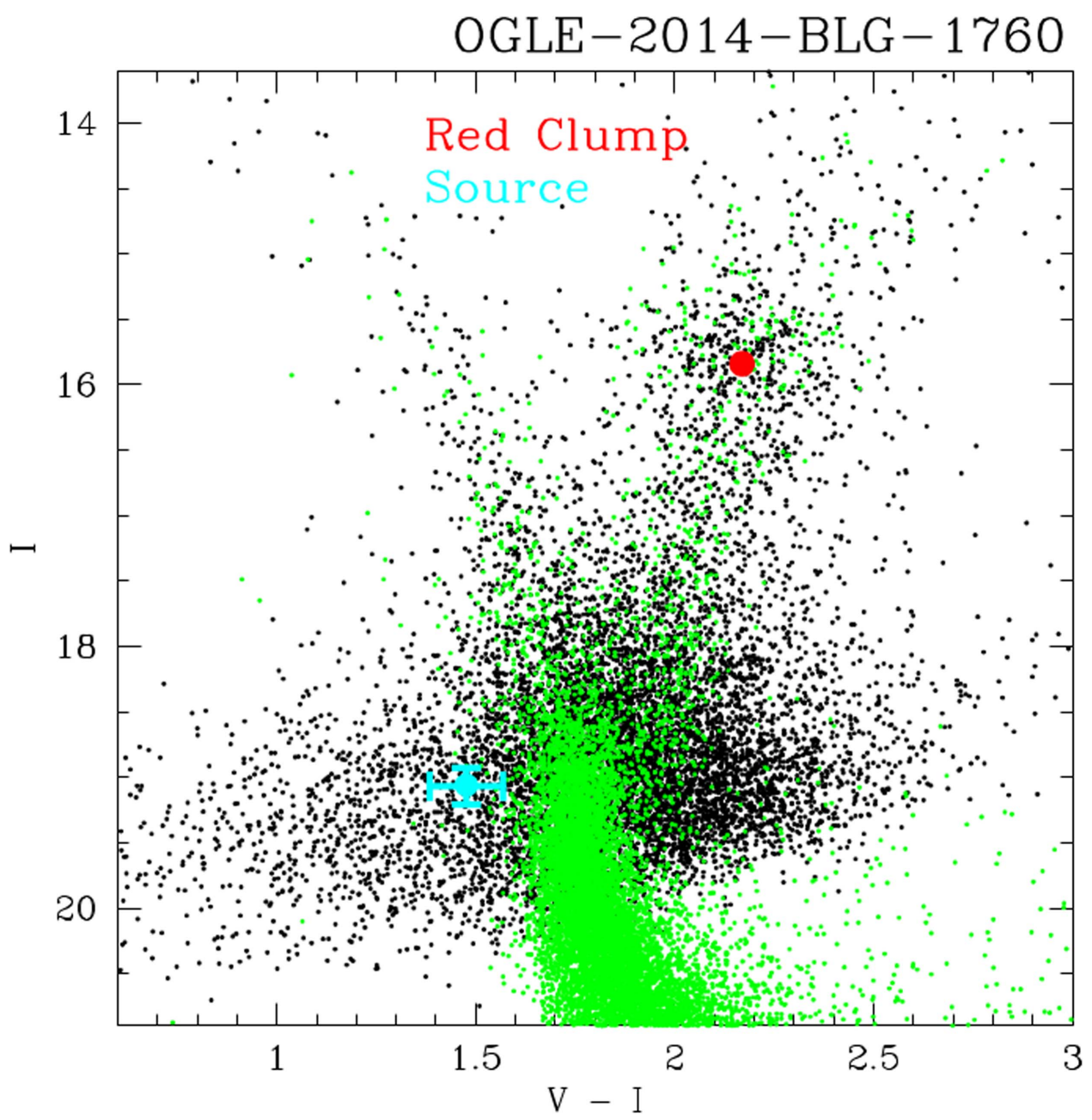

Figure 4. $(V-I, I)$ CMD of the stars in the OGLE-III catalog (Szymański et al. 2011b) within 140" of OGLE-2014-BLG-1760. The red spot indicates the red clump centroid, and the cyan spot indicates the source magnitude and color with error bars. The green points show the HST CMD of Baade's window transformed to have the same red clump centroid as this field. The source star is slightly bluer than the HST main-sequence stars. This might be explained by a young, blue source in the Milky Way disk on the far side of the bulge.

$\left(E(V-I), A_{I}\right)_{\mathrm{RC}}=(1.14,1.45)$. The extinction to the source star is assumed to be same as the average extinction for the red clump stars within $140^{\prime \prime}$ since most of the extinction is thought to be in the foreground. Thus, the extinctioncorrected source color and brightness are $(V-I, I)_{\mathrm{S}, 0}=$ $(0.34,17.62)$.

The source radius is calculated from the dereddened source magnitude and color using the following formula, obtained using a similar technique to that described in Boyajian et al. (2014):

$$
\underset{10}{\log }\left(2 \theta_{*}\right)=0.50141358+0.41968496(V-I)-0.2 I .
$$

For the dereddened source color of $V-I=0.34$ and magnitude $I=17.62$, the calculated source radius is $\theta_{*}=$ $(6.57 \pm 0.11) \times 10^{-4}$ mas. The relative proper motion and Einstein radius are calculated from

$$
\begin{gathered}
\mu_{\mathrm{rel}}=\frac{\theta_{*}}{t_{\star}} \\
\theta_{E}=\theta_{*} \times \frac{t_{E}}{t_{\star}} .
\end{gathered}
$$

Table 3

Lens System Parameters

\begin{tabular}{lcr}
\hline \hline Parameter & Units & $\begin{array}{c}\text { Mean Values and } \\
\text { Uncertainties }\end{array}$ \\
\hline Host-star mass, $M_{*}$ & $M_{\odot}$ & $0.51_{-0.28}^{+0.44}$ \\
Planet mass, $m_{\mathrm{P}}$ & $M_{J}$ & $0.56_{-0.26}^{+0.34}$ \\
Host-star-planet 2D separa- & $\mathrm{au}$ & $1.75_{-0.33}^{+0.34}$ \\
$\quad$ tion, $a_{\perp}$ & $\mathrm{kpc}$ & $6.86 \pm 1.11$ \\
Lens distance, $D_{\mathrm{L}}$ & Cousins $I$ & $23.42_{-2.92}^{+1.89}$ \\
Lens magnitude, $I_{\text {Lens }}$ & $H$ & $20.80_{-2.31}^{+1.54}$ \\
Lens magnitude, $H_{\text {Lens }}$ & & \\
\hline
\end{tabular}

Since the best-fit model has $t_{\mathrm{E}}=15.87$ days and $t_{*}=0.04$ days, we find

$$
\begin{gathered}
\mu_{\text {rel }}=6.55 \pm 1.12 \text { mas yr }^{-1} \\
\theta_{\mathrm{E}}=0.29 \pm 0.05 \text { mas. }
\end{gathered}
$$

There is a $5 \%$ uncertainty in the source-radius relation (Equation (5)). We assume 1\%-2\% error in the calibration relations (Equations (3) and (4)). Also, the uncertainty in $\mu_{\text {rel }}$ is determined from the average over all MCMC fits with the 

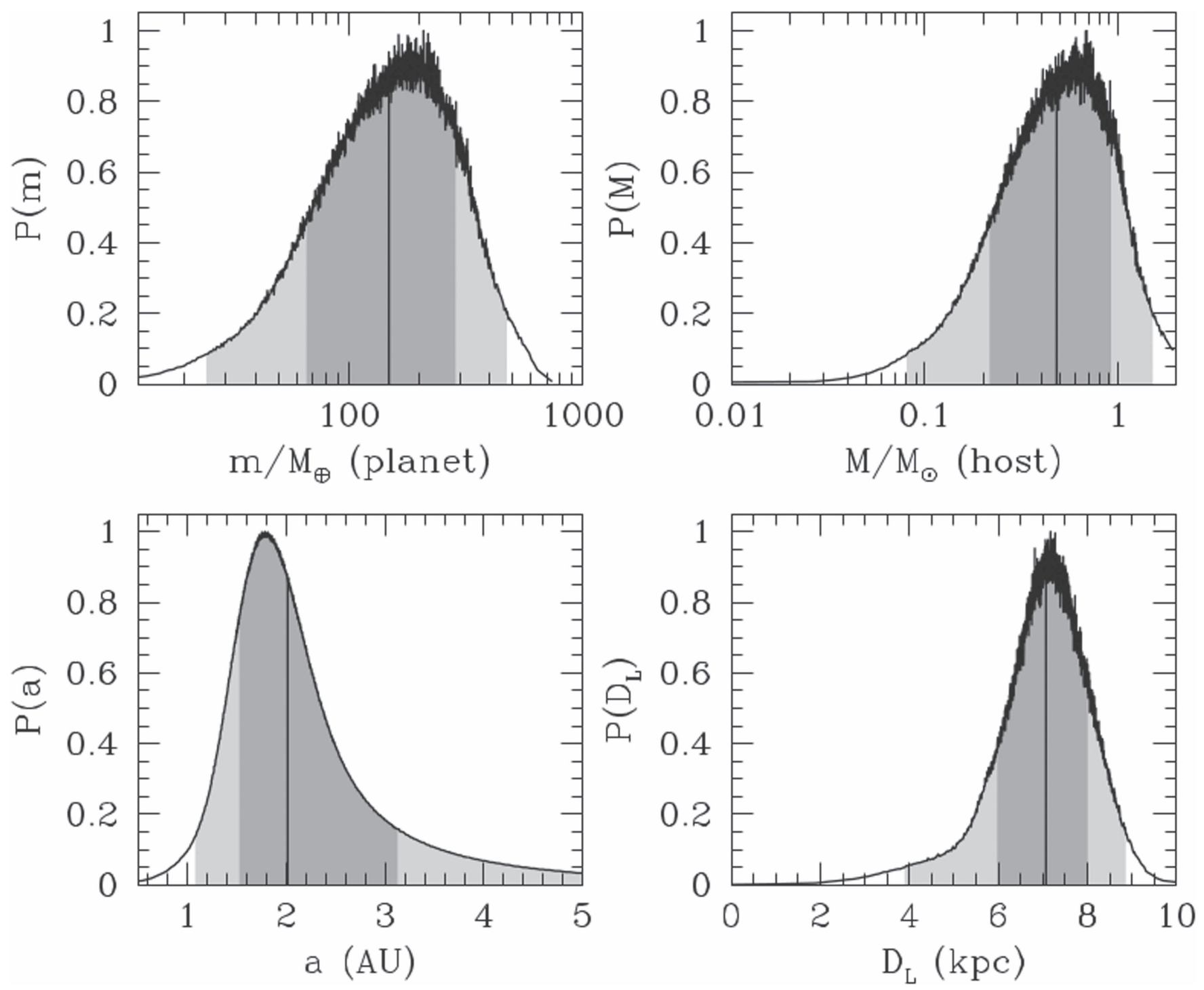

Figure 5. Probability distribution of planet mass, host-star mass, planet-host-star projected separation, and distance to the lens system, derived using Bayesian statistical analysis. The central $68.3 \%$ of the distribution is shaded dark gray, and the remaining central $95.4 \%$ of the distribution is shaded light gray. The vertical black line marks the median of the probability distribution of respective parameters.

source star distributed across $5-12 \mathrm{kpc}$ following a standard Galactic model (discussed more in Section 5).

\section{LENS PROPERTIES AND DISCUSSION}

If we assume that all stars and brown dwarfs have an equal probability of hosting a planet with the measured properties, then we can perform a Bayesian analysis to estimate the lens system properties using the technique from Bennett et al. (2014). We ran an MCMC run with about 180,000 links to obtain the posterior distributions presented in Table 3 and Figure 5. These distributions are based on the source-star brightness, color, and the radius calculated in Section 4. It is also assumed that the source star is a main-sequence bulge star distributed across 5-12 kpc following a standard Galactic model (Bennett et al. 2014). For each MCMC fit, the source distance is picked randomly from this distribution. From emperical mass-luminosity relations (Henry \& McCarthy 1993; Henry et al. 1999; Delfosse et al. 2000) and massdistance relations, we estimated the lens distance, host-star and planet mass, host-star brightness, and host-star and planet projected separation using a method similar to that used by Batista et al. (2015) and Bennett et al. (2015). Then, we calculated mean, median, and posterior distributions for each parameter from all of these MCMC fits, as seen in Table 3 and Figures 5 and 6. The mass of the host star (lens star) is approximately determined to be $M_{*}=0.51_{-0.28}^{+0.44} M_{\odot}$, so it could be an $\mathrm{M}, \mathrm{K}$, or $\mathrm{G}$ star. The $1 \sigma$ range of the planet mass, $m_{\mathrm{p}}=0.56_{-0.26}^{+0.34} M_{J}$, spans the range from the mass of Saturn to that of Jupiter. The distance to the lens system is more precisely determined, due to the relatively small angular Einstein radius, $\theta_{\mathrm{E}}=0.29 \pm 0.05$ mas. Our analysis predicts a lens system distance of $D_{L}=6.86 \pm 1.11 \mathrm{kpc}$. This implies that the lens system is very likely to be in the Galactic bulge.

Penny et al. (2016) argue that the published planetary microlensing events show a dearth of planets orbiting Galactic bulge stars. This OGLE-2014-BLG-1760L lens system would seem to be a counterexample, along with a number of other planetary microlens systems, such as OGLE-2005-BLG-380L (Beaulieu et al. 2006), OGLE-2008-BLG-092L (Poleski 

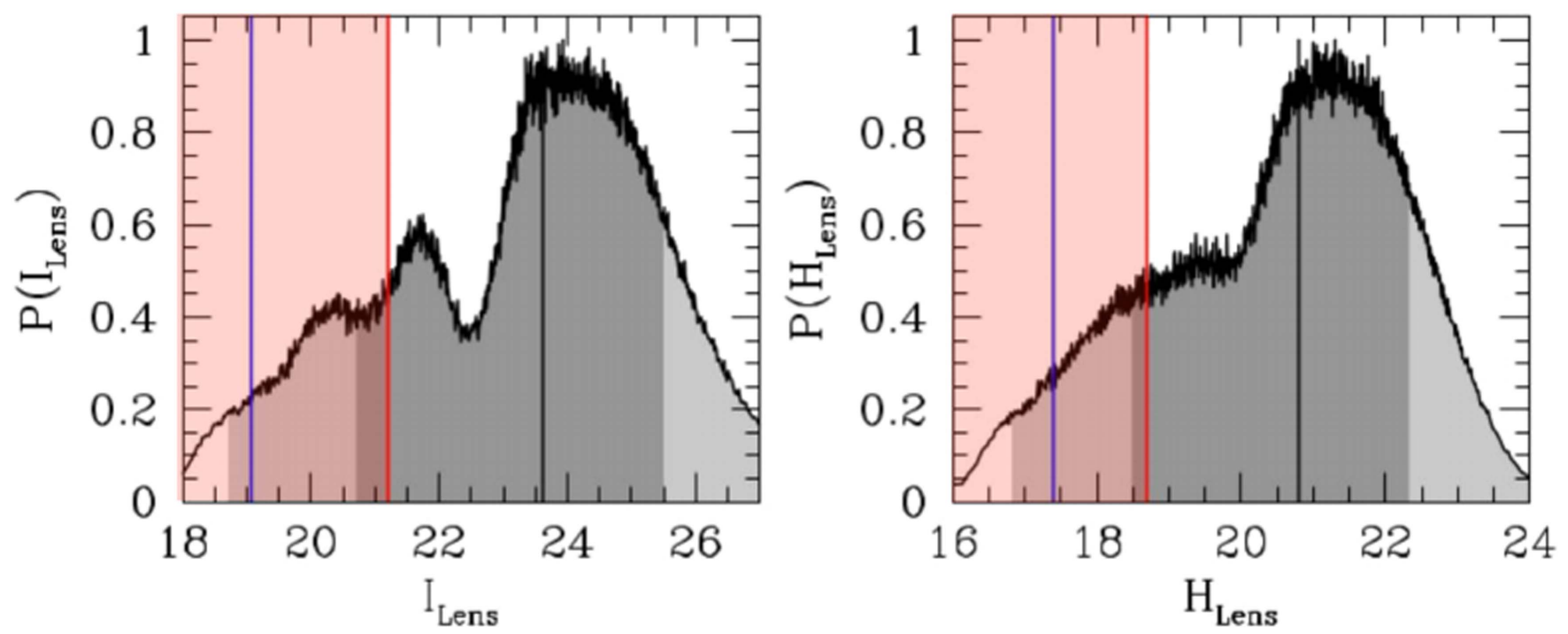

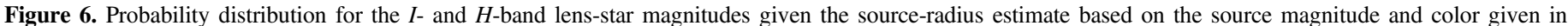

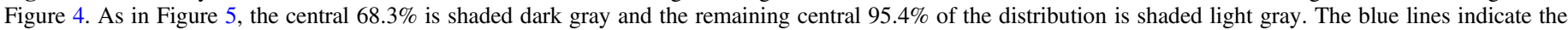

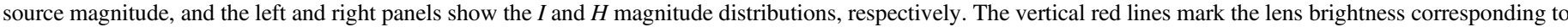

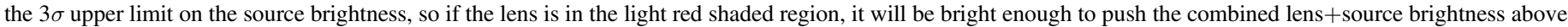

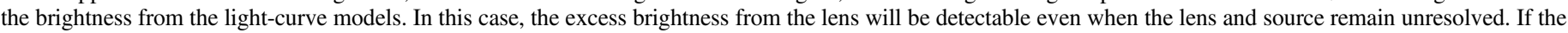

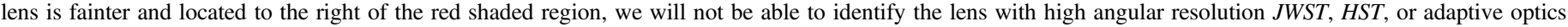
observations until the lens star begins to separate from the source 6-8 yr after peak magnification.

et al. 2014), MOA-2008-BLG-310L (Janczak et al. 2010), OGLE-2008-BLG-355L (Koshimoto et al. 2014), MOA-2011BLG-353L (Rattenbury et al. 2015), and MOA-2011-BLG293L (Yee et al. 2012; Batista et al. 2014). Actually, the problem with the Penny et al. (2016) analysis is pretty easy to understand. The Einstein radius crossing times $\left(t_{\mathrm{E}}\right)$ for bulge events are significantly smaller than the $t_{\mathrm{E}}$ values for disk events, but the detection efficiencies for microlensing events and planetary signals are substantially higher for events with large $t_{\mathrm{E}}$ values. The statistical analysis of Suzuki et al. (2016) shows that the planet detection efficiency weighted median $t_{\mathrm{E}}$ value is $\approx 42$ days, while the efficiency-corrected median is $t_{\mathrm{E}}$. This effect occurs for high-magnification events (Gould et al. $2010 \mathrm{~b}$ ) because high magnification is much easier to predict when $t_{\mathrm{E}}$ is large, and for low-magnification events (Suzuki et al. 2016) because the planetary signal duration (for fixed $q$ ) is proportional to $t_{\mathrm{E}}$. This effect can be accounted for with accurate detection efficiency calculations, but Penny et al. (2016) use a detection efficiency calculation for an advance ground-based survey that is much more sensitive than any ground-based survey undertaken to date. One of us (Bennett 2004) has previously studied a variety of ground-based microlensing surveys with a wide range of telescope options, and these simulations show that this detection efficiency bias with $t_{\mathrm{E}}$ is much stronger with the less capable surveys that can approximate the sensitivity of the observing programs that have discovered the published events.

It should also be noted that the color of the source star $V-I=1.48 \pm 0.08$ implies that this star is bluer than and only marginally consistent with the bulge main sequence shown in Figure 4. This source star could be a "blue straggler" (Sandage 1953), which is an old main-sequence, bright blue star that probably formed from a merger of two smaller stars (Ferraro et al. 2006). It could also be possible that this field has a larger fraction of bluer, metal-poor stars than the HST Baade's window stars plotted in Figure 4. Another possibility is that this star is a relatively young blue star that resides in the Galactic disk on the far side of the bulge with minimal additional extinction between the bulge and this source star. If so, HST observations taken separated by $2 \mathrm{yr}$ could reveal the characteristic source star proper motion of a far-side disk star. The results and estimates of the planetary system presented in this paper are valid if the source is a blue straggler or a blue main-sequence bulge star. If the source is a blue star in the Galactic disk behind the bulge, then the source distance will be $11 \mathrm{kpc}$ or higher and the lens is going to be more likely in the bulge. In the mass-distance relation $M_{\mathrm{L}}=\frac{c^{2}}{4 G} \frac{\theta_{E}^{2}}{\left(1 / D_{L}\right)-\left(1 / D_{S}\right)}$, Einstein angular radius $\theta_{\mathrm{E}}$ is derived from the source radius, $t_{\star}$ and $t_{\mathrm{E}}$ (see Section 4), which are already constrained by the source color and the microlensing light-curve data. So $\theta_{E}$ is also indirectly restricted. As the lens distance $D_{L}$ approaches $\sim 7 \mathrm{kpc}$ and the source distance $D_{S}$ becomes larger than $11 \mathrm{kpc}$, following the above mass-distance relation the lens mass drops. With planet/host-star mass ratio being constrained by the planetary signal, the planet system also becomes smaller and approaches Saturn analog planet mass. Since the Galactic coordinates of the source, $(l, b)=1.3186,-2.2746$, are pretty close to the nominal WFIRST exoplanet microlensing survey fields (Spergel et al. 2015), understanding the source distance distribution in this area of the bulge is important for WFIRST.

The probability distribution for the brightness of the lens star in $I$ and $H$ bands is shown in Figure 6. The blend magnitude from the best fit is found to be $I_{\mathrm{bl}}=17.94$, which is brighter than the lens-star magnitude predicted in Table 3 and Figure 6. Since the lens-source relative proper motion is

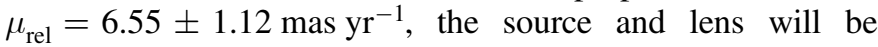
separated by $\sim 1 H S T$ pixel $(39.6 \pm 6.2$ mas) by 2020 . If the lens system is observed directly in high-resolution images, then the brightness measurement of the lens leads to the precise calculation of the mass and distance to the lens system (Bennett et al. 2006, 2015; Batista et al. 2015). But in the $I$ band, the 
source star is brighter than the median prediction for the lens by $\sim 4$ mag (see Figure 6), which makes it difficult to observe the lens in $I$ band with the high-resolution follow-up images unless the lens and source are completely resolved. The extinctioncorrected $(I-H)$ color of the source is obtained from $(V-I)$ color using Kenyon \& Hartmann (1995). The $H$-band extinction $A_{H}$ is also obtained from $A_{I}$ and $A_{V}$ using the Cardelli et al. (1989) formula. From the $I$-band magnitude, the $(I-H)$ color, and the $H$-band extinction, $A_{H}$, the $H$-band magnitude of the source is calculated to be $H_{S}=17.96$. From Figure 6, we see that the $H$-band magnitudes of the source and median prediction for the lens differ by $\sim 2.0-2.5 \mathrm{mag}$. The uncertainty in the source brightness is measured from the Markov chain links. We also include the uncertainty in the source color and magnitude. There is 5\% uncertainty in the color conversion from the extinction-corrected $(V-I)$ to $(I-H)$. The red lines in Figure 6 are such that if the lens is at least as bright as the red lines (i.e., it lies in the red shaded region), then lens + source brightness is brighter than the $3 \sigma$ upper limit on the source brightness. This would mean that the lens could be detected when it is still unresolved from the source. Since most of the lens flux histogram lies to the right of the red shaded region, the lens is likely to be too faint to be detected in high-resolution images unless lens and source are partially resolved. Since the relative lens-source proper motion

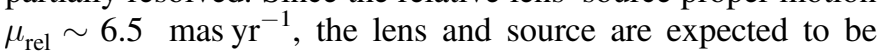
resolvable by the James Webb Space Telescope (JWST), HST, or adaptive optics imaging some 6-8 yr after peak magnification, in 2020-2022.

Suzuki et al. (2016) derived a mass ratio function from planets detected in MOA-II survey data and discovered a break in the mass ratio function at $q \sim 10^{-4}$. The OGLE-2014-BLG$1760 \mathrm{Lb}$ planetary mass ratio of $q=(8.6 \pm 0.9) \times 10^{-4}$ is above the mass ratio break $q_{\mathrm{br}} \sim 10^{-4}$. This is close to the Jupiter/Sun mass ratio, but the host star probably has a mass of $M_{*} \lesssim M_{\odot}$, so this planet is probably a low-mass gas giant, like Saturn. Follow-up observations with JWST, HST, or adaptive optics in 2020-2022 should be able to measure the lens brightness and determine the planetary mass and distance using the methods of Bennett et al. (2006, 2007, 2015), and Batista et al. (2015). Later with WFIRST (Spergel et al. 2015), a similar kind of study will provide the statistics needed to determine the planetary mass function as a function of the host-star mass and distance.

A.B., D.P.B., and D.S. were supported by NASA through grants NASA-NNX12AF54G and NNX13AF64G. I.A.B. and P.Y. were supported by the Marsden Fund of Royal Society of New Zealand, contract no. MAU1104. N.J.R. was supported through the Royal Society of New Zealand Rutherford Discovery Fellowship. A.S., M.L., and M.D. were supported through the Royal Society of New Zealand. T.S. received support from JSPS23103002, JSPS24253004, and JSPS26247023. The MOA project received grants from JSPS25103508 and 23340064. The OGLE project received funding from the National Science Centre, Poland, grant MAESTRO 2014/14/A/ST9/00121 to A.U. The OGLE team thanks Profs. M. Kubiak and G. Pietrzyński, former members of the OGLE team, for their contribution to the collection of the OGLE photometric data over the past years. A.F. was supported by the Astrobiology Project of the Center for Novel Science Initiatives (CNSI), National Institutes of Natural Sciences (NINS) (Grant Number AB261005). D.M.B. was supported by NPRP grant X-019-1-006 from the Qatar National Research Fund (a member of the Qatar Foundation). This work makes use of observations from the LCOGT network, which includes three SUPAscopes owned by the University of St Andrews. The RoboNet program is an LCOGT Key Project using time allocations from the University of St Andrews, LCOGT, and the University of Heidelberg, together with time on the Liverpool Telescope through the Science and Technology Facilities Council (STFC), UK. This research has made use of the LCOGT Archive, which is operated by the California Institute of Technology, under contract with the Las Cumbres Observatory. We thank Prof. Andrew Gould and the $\mu$ FUN team for allowing us to use their data and acknowledge their hard work and contribution in collecting the $\mu \mathrm{FUN}$ data.

\section{REFERENCES}

Alard, C. 2000, A\&AS, 144, 363

Alard, C., \& Lupton, R. H. 1998, ApJ, 503, 325

Albrow, M. D., Horne, K., Bramich, D. M., et al. 2009, MNRAS, 397, 2099

Alcock, C., Allsman, R. A., Alves, D., et al. 1995, ApJ, 454, 125

Batista, V., Beaulieu, J.-P., Bennett, D. P., et al. 2015, ApJ, 808, 170

Batista, V., Beaulieu, J.-P., Gould, A., et al. 2014, ApJ, 780, 54

Beaulieu, J.-P., Bennett, D. P., Fouqué, P., et al. 2006, Natur, 439, 437

Bennett, D. P. 2004, in ASP Conf. Proc. 221, Extrasolar Planets: Today and Tomorrow, ed. J.-P. Beaulieu, A. Lecavelier des Etangs, \& C. Terquem (San Francisco, CA: ASP), 59

Bennett, D. P. 2010, ApJ, 716, 1408

Bennett, D. P., Anderson, J., Bond, I. A., Udalski, A., \& Gould, A. 2006, ApJ, 647, L171

Bennett, D. P., Anderson, J., \& Gaudi, S. B. 2007, ApJ, 660, 781

Bennett, D. P., Batista, V., Bond, I. A., et al. 2014, ApJ, 785, 155

Bennett, D. P., Bhattacharya, A., Anderson, J., et al. 2015, ApJ, 808, 169

Bennett, D. P., Rhie, S., et al. 2010, ApJ, 713, 837

Bennett, D. P., \& Rhie, S. H. 1996, ApJ, 472, 660

Bennett, D. P., Sumi, T., Bond, I. A., et al. 2012, ApJ, 757, 119

Bensby, T., Adén, D., Melendez, J., et al. 2011, A\&A, 533, A134

Bessell, M. S., \& Brett, J. M. 1988, PASP, 100, 1134

Bond, I. A., Abe, F., Dodd, R. J., et al. 2001, MNRAS, 327, 868

Boyajian, T. S., van Belle, G., \& von Braun, K. 2014, AJ, 147, 47 Bramich, D. M. 2008, MNRAS, 386, L77

Cardelli, J. A., Clayton, G. C., \& Mathis, J. A. 1989, ApJ, 345, 245 Cassan, A., Kubas, D., Beaulieu, J.-P., et al. 2012, Natur, 481, 167 Claret, A. 2000, A\&A, 363, 1081

Delfosse, X., Forveille, T., Ségransan, D., et al. 2000, A\&A, 364, 217

Ferraro, F. R., Sabbi, E., Gratton, R., et al. 2006, ApJL, 647, L53

Gaudi, S., Bennett, D. P., Udalski, A., et al. 2008, Sci, 319, 927

Gould, A. 1992, ApJ, 392, 442

Gould, A., Dong, S., Bennett, D. P., et al. 2010a, ApJ, 710, 1800

Gould, A., Dong, S., Gaudi, B. S., et al. 2010b, ApJ, 720, 1073

Gould, A., \& Loeb, A. 1992, ApJ, 396, 104

Han, C., Bennett, D. P., Udalski, A., \& Jung, Y. K. 2016, ApJ, submitted, arXiv: 1604.06533

Henry, T. J., Franz, O. G., Wasswerman, L. H., et al. 1999, ApJ, 512, 864 Henry, T. J., \& McCarthy, D. W., Jr. 1993, AJ, 106, 773

Holtzman, J. A., Watson, A. M., Baum, W. A., et al. 1998, ApJ, 115, 1946 Ida, S., \& Lin, D. N. C. 2005, ApJ, 626, 1045

Janczak, J., Fukui, A., Dong, S., et al. 2010, ApJ, 711, 731

Kennedy, G. M., Kenyon, S. J., \& Bromley, B. C. 2006, ApJL, 650, L139

Kenyon, S. J., \& Hartmann, L. 1995, ApJSS, 101, 117

Koshimoto, N., Udalski, A., Sumi, T., et al. 2014, ApJ, 788, 128

Lecar, M., Podolak, M., Sasselov, D., \& Chiang, E, 2006, ApJ, 640, 1115

Lissauer, J. J. 1993, ARA\&A, 31, 129

Mao, S., \& Paczyński, B. 1991, ApJL, 374, L37

Muraki, Y., Han, C., Bennett, D. P., et al. 2011, ApJ, 741, 22

Nataf, D. M., Gould, A., Fouqué, P., et al. 2013, ApJ, 769, 88

Paczyński, B. 1986, ApJ, 304, 1

Penny, M., Henderson, C. B., \& Clanton, C. 2016, arXiv:1601.02807

Poleski, R., Skowron, J., Udalski, A., et al. 2014, ApJ, 795, 42

Rattenbury, N. J., Bennett, D. P., Sumi, T., et al. 2015, MNRAS, 454, 946

Sandage, A. 1953, AJ, 58, 61

Shvartzvald, Y., Udalski, A., Gould, A., et al. 2016, MNRAS, 457, 4089 
Smith, M. C., Mao, S., \& Woźniak, P. R. 2002, MNRAS, 332, 962 Spergel, D., Gehrels, N., Baltay, C., et al. 2015, arXiv:1503.03757 Suzuki, D., Bennett, D. P., Sumi, T., et al. 2016, ApJ, accepted Szymański, M. K., Udalski, A., Soszyński, I., et al. 2011a, AcA, 60, 295 Szymański, M. K., Udalski, A., Soszyński, I., et al. 2011b, AcA, 61, 83 Tomany, A. B., \& Crotts, A. P. 1996, AJ, 112, 2872
Udalski, A. 2003, AcA, 53, 291

Udalski, A., Szymański, M., Kałużny, J., et al. 1994, AcA, 44, 227 Udalski, A., Szymański, M. K., \& Szymański, G. 2015, AcA, 65, 1 Verde, L., Reiris, H. V., \& Spergel, D. N. 2003, ApJS, 148, 195 Wozniak, P. R. 2000, AcA, 50, 421

Yee, J., Shvartzvald, Y., Gal-Yam, A., et al. 2012, ApJ, 755, 102 